\title{
Genome-wide Identification and Expression Pattern Analysis of BRI1-EMS-suppressor Transcription Factors in Tomato under Abiotic Stresses
}

\author{
Yingmei Gao, Jingkang Hu, Tingting Zhao, Xiangyang Xu, Jingbin Jiang, and Jingfu Li ${ }^{1}$ \\ Department of Horticultural Sciences, Northeast Agricultural University, Harbin 150030, \\ Heilongjiang, China
}

\begin{abstract}
AdDitional INDEX words. BES1, expression characteristics, gene structure, phylogeny, Solanum lycopersicum, stress resistance

ABSTRACT. BRI1-EMS-suppressor 1 (BES1) is a transcription factor (TF) that functions as a master regulator of brassinosteroid (BR)-regulated gene expression. Here, we provide a complete overview of Solanum lycopersicum BES1 (SLB) genes, including phylogeny, gene structure, protein motifs, chromosome locations and expression characteristics. Through bioinformatic analysis, we compared the sequences of SLB genes, arabidopsis (Arabidopsis thaliana) genes, and chinese cabbage (Brassica pekinensis) genes. All of the gene sequences were divided into three groups by cluster analysis. SLB genes were mapped to the eight tomato (S. lycopersicum) chromosomes. Bioinformatic analysis showed that SLB genes shares similarities with the proteins from other plants, though different species exhibit specific features. The expression patterns of SLB genes in various tissues and under different abiotic conditions were analyzed by quantitative reverse transcription polymerase chain reaction. SLB genes were found to be induced by multiple stresses, particularly salt stress, indicating that SLB genes may have important roles in the response to unfavorable environmental changes. This study provides insight into the evolution of SLB genes and may aid in the further functional identification of BES1 proteins and the response of tomato plants to different stresses.
\end{abstract}

Transcription factors are proteins that bind to a specific nucleotide sequence and play a central role in gene regulation by activating or repressing the transcription of target genes. TFs can also activate a wide array of defense mechanisms in plants suffering from biotic and abiotic stresses (Century et al., 2008). BRI1-EMS-suppressor 1 is a new class of TFs that bind to and activate the promoters of BR genes (Yin et al., 2005). BRs regulate many plant growth and developmental processes, such as vascular development, cell elongation, stress responses, and senescence (Liand Chory, 1999). Extensive research has revealed the roles of BES1 TFs in plant growth, stress responses, and development (Guo et al., 2013; Ryu et al., 2010; Yin et al., 2005). Activated BES1 leads to the phosphorylation of TFs and other signaling components that regulate expression of downstream genes (Yin et al., 2005). Moreover, BES1 functions with BES1-interacting Myc-like 1 (BIM1), a basic helix-loop-helix TF, to synergistically bind to E box sequences to activate several BR-induced gene promoters (Yin et al., 2005). BRs have been shown to act as key components of the signaling pathways that mediate responses to abiotic and biotic stresses such as drought, hypoxia, and osmotic stress (Fujioka and Yokota, 2003). BES1 is phosphorylated and appears to be destabilized by the glycogen synthase kinase-3 BRASSINOSTEROID INSENSITIVE2 (BIN2), a negative regulator of the BR pathway (Yin et al., 2002). It has been

Received for publication 1 Dec. 2017. Accepted for publication 3 Jan. 2018. This study was funded by the National Key R\&D Program of China (2017YFD0101900), the China Agriculture Research System (CARS-25-A15), and the Breeding of New Staple Vegetable Varieties of Heilongjiang Province (GA15B103).

I thanks the help of key Laboratory of Biology and Genetic Improvement of Horticultural Crops (Northeast Region), Ministry of Agriculture/Northeast Agricultural University, Harbin, Heilongjiang, People's Republic of China.

${ }^{1}$ Corresponding author. E-mail: lijingfu@yahoo.com. revealed that the BES1 protein contains a putative nuclear localization sequence, followed by a highly conserved aminoterminal domain $(\mathrm{N})$ shared among BES1 and family members, a BIN2 phosphorylation domain (P), a PEST motif, and a carboxyl-terminal domain (C) (Wu et al., 2016; Yin et al., 2005). More recent studies have shown that BES1 regulates the localization of BR receptor BRL3 within the provascular tissue of the arabidopsis primary root (Salazarhenao et al., 2016).

Tomato is an important crop grown in many areas around the world. In the present study, a genome-wide bioinformatic analysis was conducted to provide a relatively complete profile of the SLB gene family. The chromosomal localizations, gene structures, and conserved motifs in the promoter regions of these genes were analyzed, and used quantitative reverse transcription polymerase chain reaction (qRT-PCR) to analyze the expression of nine SLB genes in various tissues and under different abiotic stresses. In this study, we identified nine SLB genes by downloading their sequences and analyzing their gene structures, chromosomal locations, gene duplicates, and evolutionary mechanisms. We also discuss the kinetics of the corresponding transcription patterns in response to cold, drought, and salt (abiotic stress), as estimated by qRT-PCR. We report that some SLB genes are connected to tissue development and some gene expression was controlled by different abiotic stress reactions. These findings provide an important starting point for future research on the biological functions of the BES1 family in tomato.

\section{Materials and Methods}

Plant growth conditions and stress treatments. For RNA isolation, the tomato cultivar Moneymaker [World Vegetable Center (AVRDC), Shanhua, Taiwan] was grown as the experimental plant material at the Northeast Agricultural University 
Experimental Station (Harbin, China). Seedlings were grown in a mixture of perlite:vermiculite:plant ash (1:6:2) in a growth room under fluorescent lighting $\left(200 \mu \mathrm{E} \cdot \mathrm{m}^{-2} \cdot \mathrm{s}^{-1}\right)$ at 22 to $24{ }^{\circ} \mathrm{C}$, with $60 \%$ relative humidity and a $14 / 10$-h light/dark cycle ( $\mathrm{Li}$ et al., 2015). To investigate organ-specific expression profiles, samples of five types of organs (fibrous roots, young stems, young leaves, petals, and mature fruit) were collected from adult plants. Three samples of the tomato tissues were obtained from different plants. Two-month-old seedlings were used for all abiotic treatments. Seedlings at the four-leaf stage were put into growth chambers set at $4{ }^{\circ} \mathrm{C}$ under the fluorescent lighting $\left(200 \mu \mathrm{E} \cdot \mathrm{m}^{-2} \cdot \mathrm{s}^{-1}\right)$ at 22 to $24{ }^{\circ} \mathrm{C}$ and a $14 / 10$-h light/dark cycle with $60 \%$ relative humidity for cold treatment. Before the drought and salinity treatments, the seedlings were transferred to Hoagland's nutrient solution for $24 \mathrm{~h}$ (Hoaglandand Arnon, 1937), under the fluorescent lighting $\left(200 \mu \mathrm{E} \cdot \mathrm{m}^{-2} \cdot \mathrm{s}^{-1}\right)$ at 22 to $24{ }^{\circ} \mathrm{C}$ and a $14 / 10$-h light/dark cycle $60 \%$ relative humidity and then irrigated with $200 \mathrm{mmol} \cdot \mathrm{L}^{-1}$ $\mathrm{NaCl}$ and $15 \%$ polyethylene glycol (PEG), respectively, for $24 \mathrm{~h}$. Each treatment had three to five seedlings, and all the treatments had three biological replicates. Young leaf samples were collected at different time points $(1.5,3,6,12$, and $24 \mathrm{~h})$ and then immediately frozen in liquid nitrogen and stored at $-80{ }^{\circ} \mathrm{C}$ before RNA extraction.

IDENTIFICATION OF SLB Genes. Searches of the nucleotide and protein sequences of SLB genes were conducted in Sol Genomics Network (International Solanaceae Initiative, 2015). The BES1 protein sequences of arabidopsis and chinese cabbage were downloaded from The Arabidopsis Information Resource (Phoenix Bioinformatics Corporation, 2013) (Huala et al., 2001) and the BRAD database (Cheng et al., 2011). The BES1 domain (PF05687) obtained from the PFAM database was used as the query for a hidden Markov model search using the HMMER 3.0 program with a predefined threshold of $E<1 \mathrm{e}-5$ (Artimo et al., 2012; Cui et al., 2016). The molecular weight and the theoretical isoelectric point ( $\mathrm{pI}$ ) of the obtained proteins were determined using the computing $\mathrm{pI} / \mathrm{Mw}$ tool on the ExPASy server (Artimo et al., 2012).

Phylogenetic tree analyses and Sequence alignment. Multiple sequence alignments of the amino acid sequences of the BES1 proteins of tomato, arabidopsis, and chinese cabbage were generated with ClustalX 2.0 software (Larkin et al., 2007). A phylogenetic tree based on the sequence alignments was constructed using MEGA 5.0 software (Tamura et al., 2011) and the neighbor-joining method with 1000 bootstrap replicates, maximum composite likelihood.

Conserved domain and Gene STRuCture. We used the sequence analysis software DNAMAN5.0 and the online software WebLogo (Crooks et al., 2004) to analyze conserved sequences of the tomato BES1 protein. The genomic sequences of SLB genes were then retrieved, and online Gene Structure Display Server (Center for Bioinformatics, 2015) was used to decipher the architecture of the SLB genes (Bai et al., 2016).

Chromosomal Distribution. To determine the physical locations of SLB genes on chromosomes, Phytozome (Energy's Joint Genome Institute, 1997) was used to identify the initial site of each gene. MapInspect software was then used to draw images of the locations of the SLB genes (Song et al., 2014). Standards regarding gene duplication followed the criteria described by Chen et al. (2012).

RNA EXTRACTION AND REVERSE TRANSCRIPTION OF CDNA. RNA samples were extracted using the TRIzol method according to the manufacturer's instructions (Rio et al., 2010). Total RNA was treated with RNase-free DNase (TaKaRa, Dalian, China). Reverse transcription was performed with $1 \mu \mathrm{g}$ of total RNA in a $20-\mu \mathrm{L}$ volume with TransScript ${ }^{\circledR}$ RT/RI Enzyme Mix (Transgen Biotech, Beijing, China), according to the manufacturer's instructions.

Quantitative Real-time PCR analysis. The analysis was performed using the iQ5 real-time PCR platform (Bio-Rad Laboratories, Hercules, CA). Each $20-\mu \mathrm{L}$ quantitative polymerase chain reaction mixture contained $2.5 \mu \mathrm{L}$ of first-strand cDNA, $10 \mu \mathrm{L}$ of $2 \times$ FastStart Universal SYBR Green Master (Vazyme, Nanjing, China), and $0.2 \mu \mathrm{M}$ of forward and reverse primers for each gene. The reaction was initiated with a preliminary step of $95^{\circ} \mathrm{C}$ for $3 \mathrm{~min}$, followed by 40 cycles of $95^{\circ} \mathrm{C}$ for $10 \mathrm{~s}$, and $60^{\circ} \mathrm{C}$ for $30 \mathrm{~s}$. The comparative $\mathrm{Ct}$ value method was adopted to analyze relative gene expression. RNA expression levels relative to the actin gene were calculated as $2^{-\triangle \triangle \mathrm{CT}}$ values according to a previous analysis (Pfaffl, 2001). The primers used for qRT-PCR were designed according to the genomic sequences of SLB genes using Primer 5 (Jie et al., 2013) software, and the Actin 7 gene was used as an internal control (Wei et al., 2015) (Supplemental Table 1). The qRTPCR data were clustered with the Pearson correlation distance metric using the average linkage method. The results are shown as color-coded heatmaps that reflect absolute signal values. The heatmaps were plotted using OmicShare tools (Gene Denovo, 2015), a free online platform for data analysis.

\section{Results}

IDENTIFICATION AND SEQUENCE ALIGNMENT OF SLB FAMILY GENES IN TOMATO. Using the method described above, nine genes were identified as candidate BES1 genes in the tomato

Table 1. Characteristic features of BRI1-EMS-suppressor 1 (BES1) transcription factor gene family identified in tomato.

\begin{tabular}{|c|c|c|c|c|c|c|c|}
\hline Gene ID & Gene name & Amino acids (no.) & Domain size (aa) & MW (Da) & Theoretical pI & Aliphatic index & Gravy \\
\hline Solyc01g094580.2.1 & SLB1 & 695 & $69-213$ & $77,864.45$ & 5.37 & 71.41 & -0.466 \\
\hline Solyc02g063010.2.1 & SLB2 & 319 & $9-153$ & $34,474.87$ & 9.38 & 65.52 & -0.516 \\
\hline Solyc02g071990.2.1 & SLB3 & 324 & $2-142$ & $34,908.89$ & 8.14 & 57.78 & -0.606 \\
\hline Solyc04g079980.2.1 & SLB5 & 327 & $28-159$ & $34,977.19$ & 8.88 & 62.69 & -0.519 \\
\hline Solyc07g062260.2.1 & SLB6 & 315 & $2-145$ & $33,827.99$ & 9.00 & 53.68 & -0.519 \\
\hline Solyc08g005780.2.1 & SLB7 & 666 & $60-202$ & $75,255.45$ & 6.09 & 66.47 & -0.492 \\
\hline Solyc12g089040.1.1 & SLB9 & 333 & $36-171$ & $35,772.85$ & 8.85 & 56.01 & -0.637 \\
\hline
\end{tabular}

ID = identity; SLB = Solanum lycopersicum BES1 gene 8; aa = amino acids; MW = molecular weight; $\mathrm{pI}=$ isoelectric point; Gravy = grand average of hydropathy. 
genome. HMM analysis using the SMART/Pfam tool revealed that all of the deduced protein sequences contain DUF822, a conserved BES1 domain. The amino acid sequence lengths encoded by SLB genes ranges from 180 (SLB8) to 695 (SLB1). The molecular masses range from 20,389.00 (SLB8) to $77,864.45$ (SLB1) Da and the pI values of the proteins from 5.37 (SLB1) to 9.38 (SLB2). The characteristic features of the SLB gene family are shown in Table 1.

Two conserved domains were identified in the SLB genes: DUF822 and glycosyl hydrolase 14 (Fig. 1). DUF822 is also known as BES1_N, comprising the N-terminal regions of several TFs. This sequence was classified as BES1/BZR1, a plant-specific TF that cooperates with other TFs such as BIM1 to regulate BR-induced genes. Located at the N-terminus, the domain is $\approx 110-148$ amino acids. Only SLB1 and SLB7 exhibit the conserved glycosyl hydrolase 14 domain, corresponding to a family of glycoside hydrolases.

Phylogenetic analysis of SLB genes. A phylogenetic tree based on nine SLB, six arabidopsis AtBES1, and 15 chinese cabbage BrBES1 protein sequences was constructed. In this tree, the SLB gene sequences were divided into three main groups: Group I (5), Group II (10), and Group III (15) (Fig. 2). Group I contains the lowest number of BES1 sequences, including only SLB1 and SLB7, which exhibit both the DUF822 and glycosyl hydrolase 14 conserved domains. Group II is the largest tomato group, with four SLBs (SLB8 performed more similar to Group II genes than Group III after sequence alignment). Group III contains nine BrBES1, three SLB, and three AtBES1 sequences, thus constituting the family with the greatest number.

Gene STRUCTURE AND CONSERVED MOTIF IDENTIFICATION IN томато. Considering that a gene structure is a typical imprint of evolution within a gene family, we analyzed the BES1 genes of the above three plants using the tools available on the GSDS website (Bo et al., 2015; Center for Bioinformatics, 2015). The genes in each group showed similar structures and introns (Fig. 3C). As almost all SLB genes contain one or two introns except SLB1 and SLB7, each SLB sequence is divided into many segments by introns. Among the nine SLB genes, two present a 9-exon/9-intron structure and seven present a 2-exon/ 1 -intron structure. Interestingly, all BES1 genes in arabidopsis contain upstream/downstream area; all genes without upstream/downstream area belong to chinese cabbage and tomato.

The conserved motifs were analyzed using the MEME program (National Institutes of Health, 2015; Fig. 3B), and LOGOs of the protein motifs were obtained with MEME (Fig. 1). Ultimately, we identified 20 motifs (with an $E$-value cutoff <e-1.0) (Baloglu et al., 2014) (Supplemental Fig. 1). With the exception of AT1G32130, all of the sequences contain motif 1 , motif 2, and motif 14 and belong to the family of DUF822 conserved domains. Moreover, motifs 5, 11, 12, 13, 14,18 , and 19 were reshared by SLB1 and SLB7. Some of the other conserved motifs were also specifically found in Group I. The results indicated that genes from the same subfamily might share similar functions and motif compositions.

Distribution of BES1 genes ON TOMATO CHROMOSOMES. We used MapInspect software to determine the chromosomal distribution of the SLB genes. All of the SLB sequences were mapped to the eight chromosomes of tomato. Chromosome 2 was the only chromosome containing two genes (SLB1 and SLB2), whereas no SLB genes are present on chromosomes 5, 6, 9, and 11. On average, one

\section{AN-terminal (DUF822 domain)}

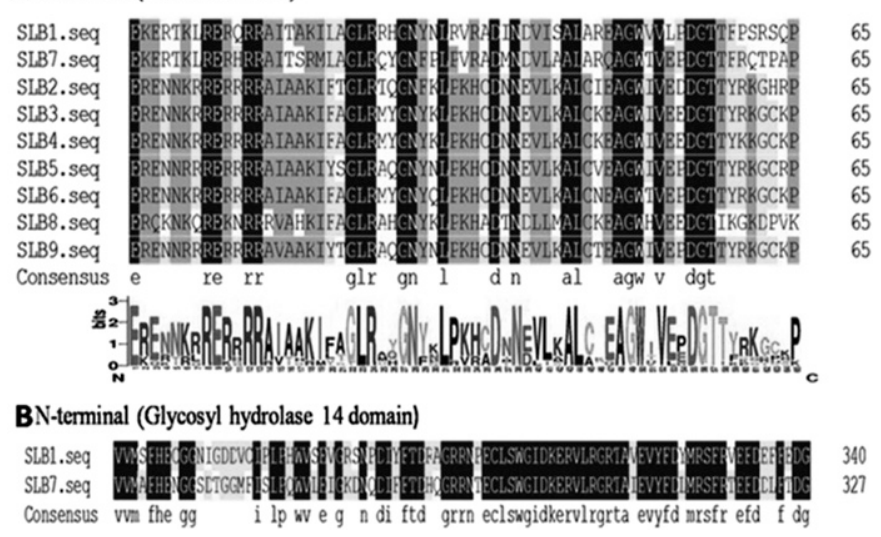

Fig. 1. Multiple alignments of the BRI1-EMS-suppressor 1 (BES1) domains from tomato amino acid and sequence logo of the DUF822 and Glycosyl hydrolase 14 domains of the tomato BES1 proteins. Conserved amino acid residues are indicated in red or blue. The height of each stack and single letters in every stack show the level of conservation at this location and the relative frequency of each amino acid, respectively.

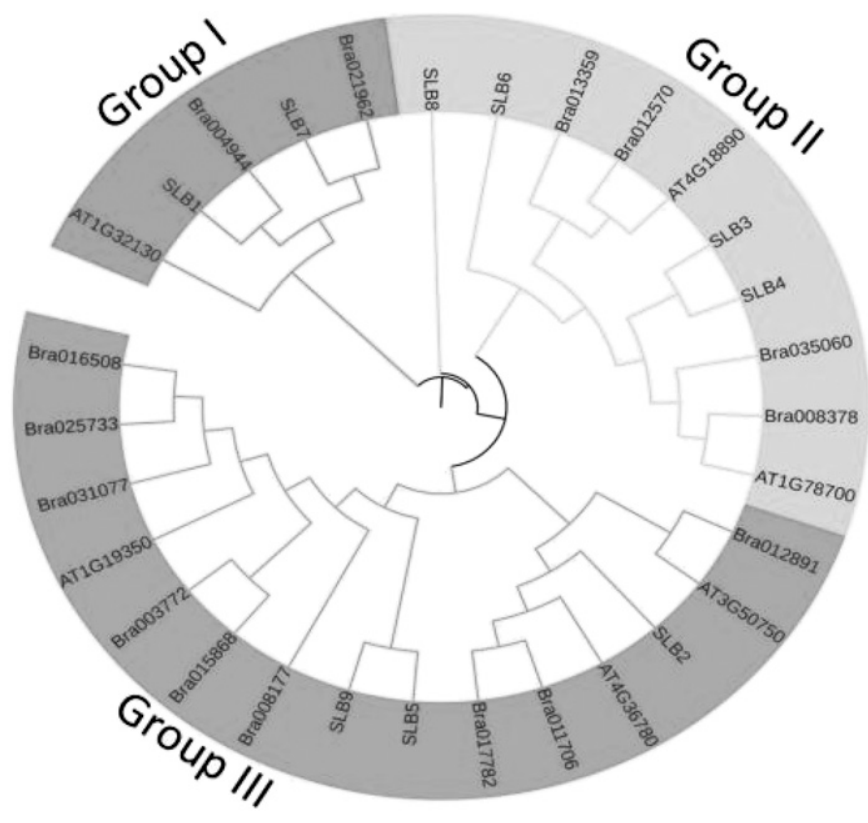

Fig. 2. Phylogenetic analysis of BRI1-EMS-suppressor 1 (BES1) family members in tomato. A neighbor-joining phylogenetic tree was generated by full-length BES1 sequences from arabidopsis, tomato, and chinese cabbage. The tree was divided into three phylogenetic subgroups designated as Group I-III marked with different color backgrounds; SLB = Solanum lycopersicum BES1, AT = Arabidposis Tair, Bra = Brassica .

SLB gene occurs every 34.3 Mb. Gene duplication has been studied in many plant species (Wang et al., 2015). In tomato, two duplicated SLB genes were identified, which were divided into two groups: SLB3 and SLB4 belonging to Group II and SLB5 and SLB9 belonging to Group III. Both pairs are the result of segmental duplications, and no tandem duplicated genes were found.

EXPRESSION PATTERnS OF SLB IN VARIOUS Tissues. To investigate the functional divergence of SLB genes, expression of all nine SLB genes was analyzed in different tissues under normal conditions by qRT-PCR (Fig. 4). With the exception of SLB2 and SLB5, all of the other genes showed high expression 


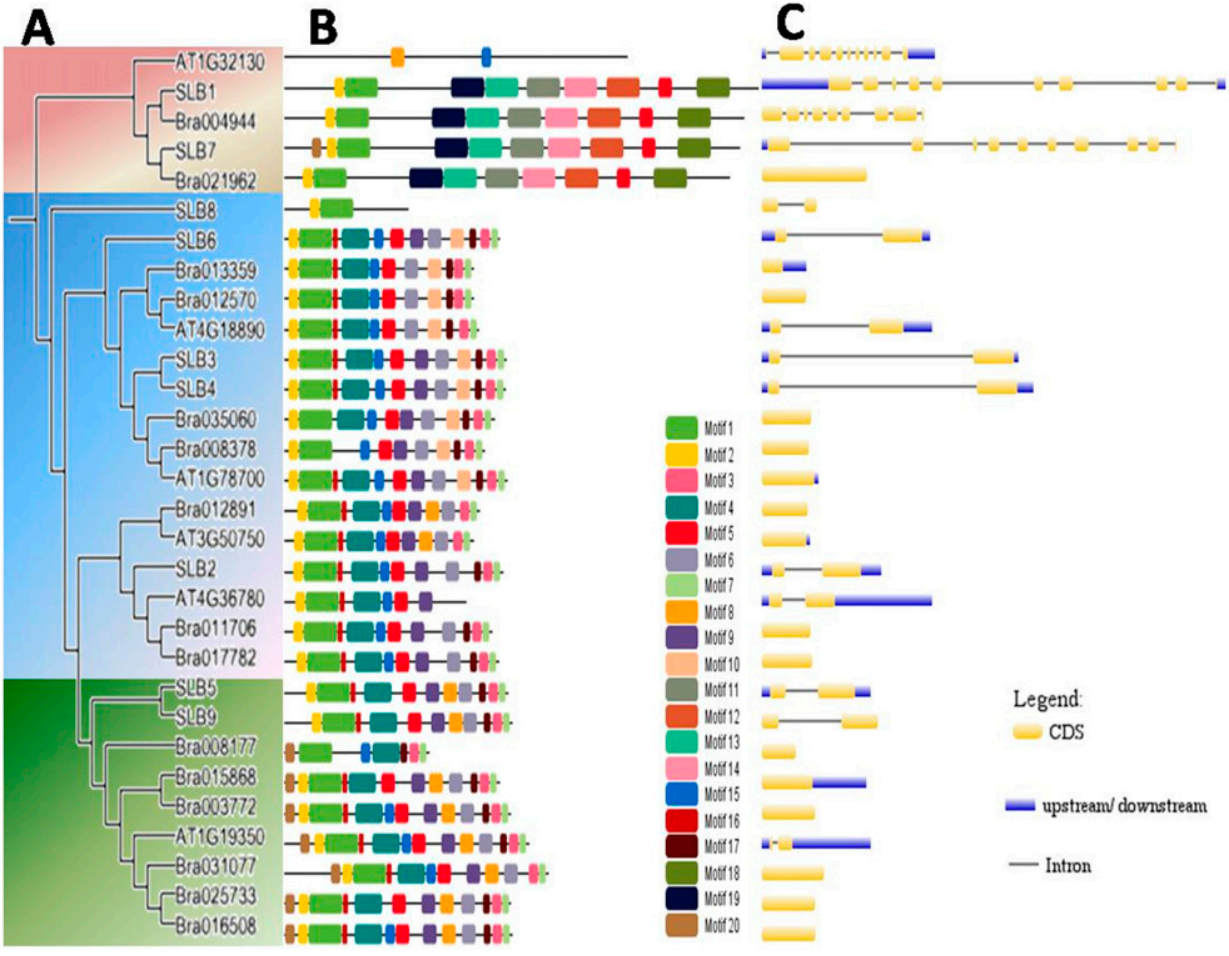

Fig. 3. Phylogenetic relationships, conserved motif compositions and gene structures of BRI1-EMS-suppressor 1 (BES1) proteins. (A) The phylogenetic tree was constructed by the full-length BES1 sequences of arabidopsis, tomato, and chinese cabbage. (B) The schematic representation of the conserved motifs in the BES1 proteins. Each motif is represented by a color box numbered at the bottom. (C) The gene structures of BES1, including introns, exons, and upstream/downstream, are represented by black lines, yellow, and blue boxes, respectively (color figure online).

highly expressed in fruit, indicating that SLB genes may not be involved in fructification.

EXPRESSION PATTERNS OF SLB UN DER D IFFER ENT ABIOTIC CONDITIONS. To investigate the role of SLB genes, all nine SLB genes were selected for evaluation by qRT-PCR of comprehensive expressional profiles under drought and cold treatments. The results showed (Fig. 5) that some SLB genes exhibit diverse gene expression patterns in response to different treatments (Fig. 5A). Under PEG treatment, some genes were downregulated, such as SLB2 and SLB5, whereas genes such as SLB1 and SLB6 were upregulated within $24 \mathrm{~h}$; moreover, SLB2 showed a downward trend as the duration of stress progressed. In addition, expression of SLB1 was upregulated by $>1.58$ fold compared with that of the control treatment after $1.5 \mathrm{~h}$ of drought stress. These results suggest that both SLB1 and -2 are closely related to drought stress but may have different mechanisms. SLB1, SLB6, SLB7, and SLB8 were downregulated under cold treatment, and SLB9 expression displayed a ten-

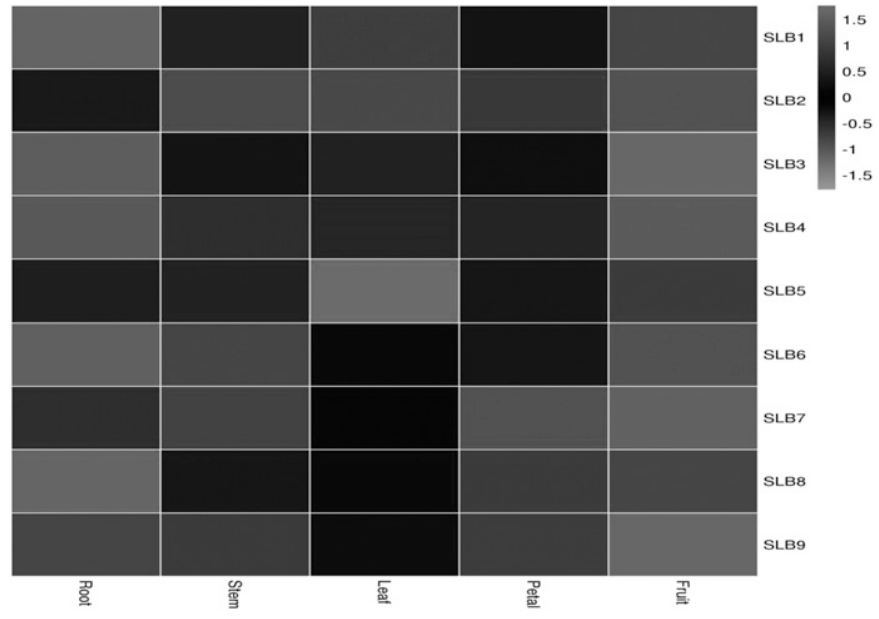

Fig. 4. Expression profiles of Solanum lycopersicum BRI1-EMS-suppressor 1 (SLB) genes in different organs/tissues using quantitative reverse transcription polymerase chain reaction analysis. All samples were run in triplicate and the data were normalized relative to the actin 7 transcript levels. The bar at the right of heat map and column represents relative expression values.

levels in roots. In the stem, only SLB2 was expressed at a high level, and both SLB4 and SLB5 presented high expression in leaves, with the latter only showing high expression in leaves. Petals were the organ which own the greatest number of genes exhibited high expression, including SLB2, SLB6 and SLB7, SLB7 showed a significantly high expression level. SLB1 genes displayed high expression levels in all other tissues, though was dency to decline initially and later increase. With the exception of SLB2, all of the other genes were significantly upregulated after 6 and $24 \mathrm{~h}$ of salt treatment. Moreover, expression of some genes was upregulated by $>100$-fold after 6 and $12 \mathrm{~h}$ of salt treatment compared with other treatment times. Thus, expression of these genes was clearly increased in response to salt treatment, indicating that they play an important role in resistance to salt stress in tomato. Interestingly, SLB2 was upregulated under both cold and $12 \mathrm{~h}$ of salt treatment. Hence, we deduce that SLB2 functions in both cold and salt stresses.

\section{Discussion}

Tomato is an important crop that is cultivated worldwide. According to PlantTFDB (Jin et al., 2017), BES1 genes have been discovered in 149 plant species in the past decade, with the number of genes in various species ranging from 2 to 42 , and 30 BES1 genes have been analyzed. Advances in molecular analyses have improved our understanding of the BES1 TF family. Wu et al. (2016) identified 15 BES1 genes in chinese cabbage and found that BES1 was present only in land plants. However, the BES1 genes of tomato have not been characterized to date, especially in response to abiotic stresses. In this study, we performed a comprehensive search for BES1 genes in the tomato genome and found nine. We used bioinformatics to analyze the BES1 TF family and the expression patterns of the encoding genes in different tissues and under different abiotic conditions.

According to our bioinformatics analysis, the tomato BES1 gene family can be divided into three groups, similar to the 


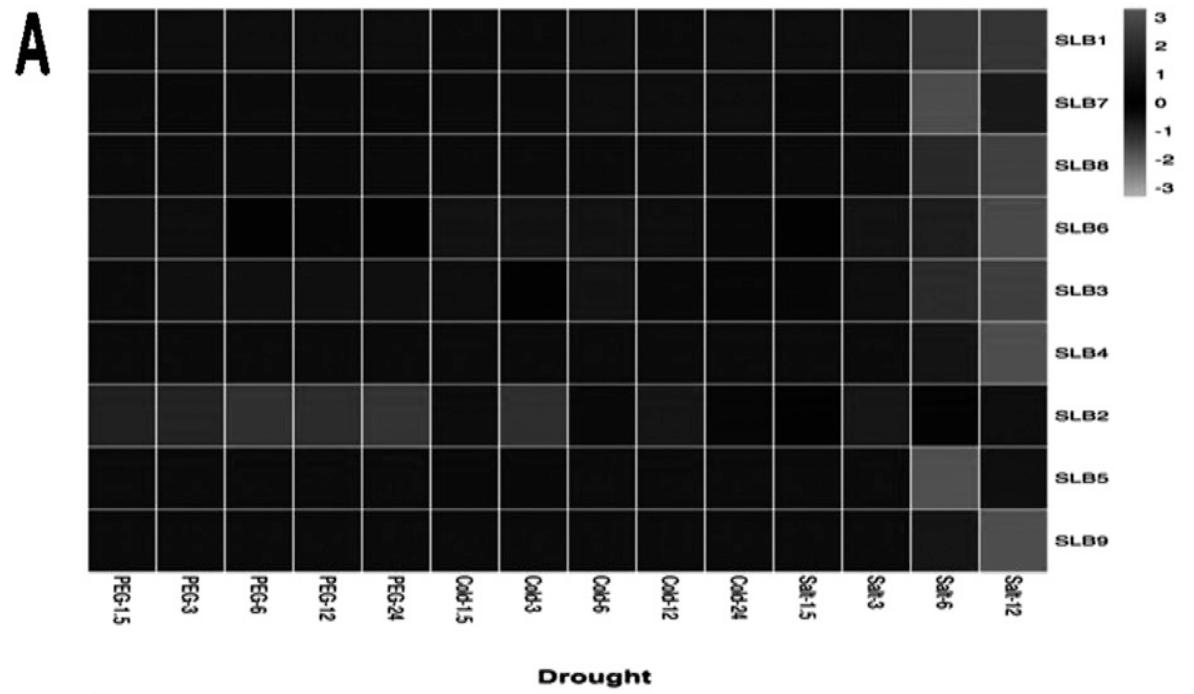

tomato BES1 genes. Two conserved domains, BES1_N and glycosyl hydrolase 14, are present in tomato BES1 TFs (Fig. 1), a finding that is similar to previous results showing that chinese cabbage BES1 contains BISON and glycosyl hydrolase 14 domains. All nine SLB genes harbor a BES1_N domain. Thus, the BES1_N type is the BES1 domain arrangement among BES1 family members in plants. Gene duplication occurs continuously during the evolution of living organisms and is considered a major cause of genome complexity and functional expansion (Magadum et al., 2013; Wei et al., 2016). Duplicated genes are highly similar in terms of their nucleotide sequence, gene structure, protein sequence, and chromosomal location. In our analysis of SLB, two pairs of putatively duplicated genes highly similar in their nucleotide sequence were discovered. For example, SLB3 on chromosome two and SLB4 on chromosome three encode proteins that are $95 \%$ identical in amino acid sequence (Fig. 6). Gene duplication can result in redundancy of gene function or generation of complementary genes. In this case, the duplicated BES1 genes may have similar or different expression patterns.

We identified expression of all nine tomato SLB genes in various tissues. Our results indicated high expression levels of SLB genes in roots but low expression levels in fruits. Espinosa-Ruiz et al. (2017) found that BR may control shoot boundaries and root meristem development in arabidopsis, which indicates that BES1 genes may be closely associated with the growth of roots and less associated with fruit development. Moreover, all nine SLB genes were examined under different stress treatments via qRT-PCR. By regulating an array of Fig. 5. Expression analysis of Solanum lycopersicum BRI1-EMS-suppressor 1 (SLB) genes under cold, drought, and salt abiotic stresses. (A) Heat map representation and hierarchical clustering of SLB genes under the stresses. (B) The relative expression ratios of SLB genes under cold, drought, and salt stresses. The relative expression levels of SLB in leaves under these stresses were quantified against the control transcript levels. The bar at the right of heat map and column represents relative expression values; $\mathrm{CK}=$ control; 1.5, 3, 6, 12, $24=$ treat time in hours; PEG = polyethylene glycol drought treatment.

categories of BES1 genes observed in arabidopsis and chinese cabbage (Wu et al., 2016). Although the SLB genes present a similar classification as arabidopsis BES1 genes, their numbers in each group were different, proving that different plants show multiple duplications. The comparison of the homologs between species showed that SLB1 is homologous to Bra004944 (B. pekinensis), and SLB7 is homologous to Bra021962, which identified a closer relationship for tomato with chinese cabbage than with arabidopsis. For example, based on the patterns of gene expression, which were closely connected with gene function, we can predict the functions of genes, TFs are crucial for controlling different mechanisms (Broun, 2004; Zhao et al., 2012). According to previous studies, arabidopsis and chinese cabbage BES1 genes are associated with stress responses (Wu et al., 2016). As abiotic stresses, such as drought, are causing increasing injury to crop production and quality, a thorough understanding of the molecular mechanisms involved in plant stress tolerance has become pivotal for the development of new strategies and technologies related to the increasing demand of agricultural production (Chen et al., 2015). The results of the present study revealed that almost all SLB genes were induced to some 


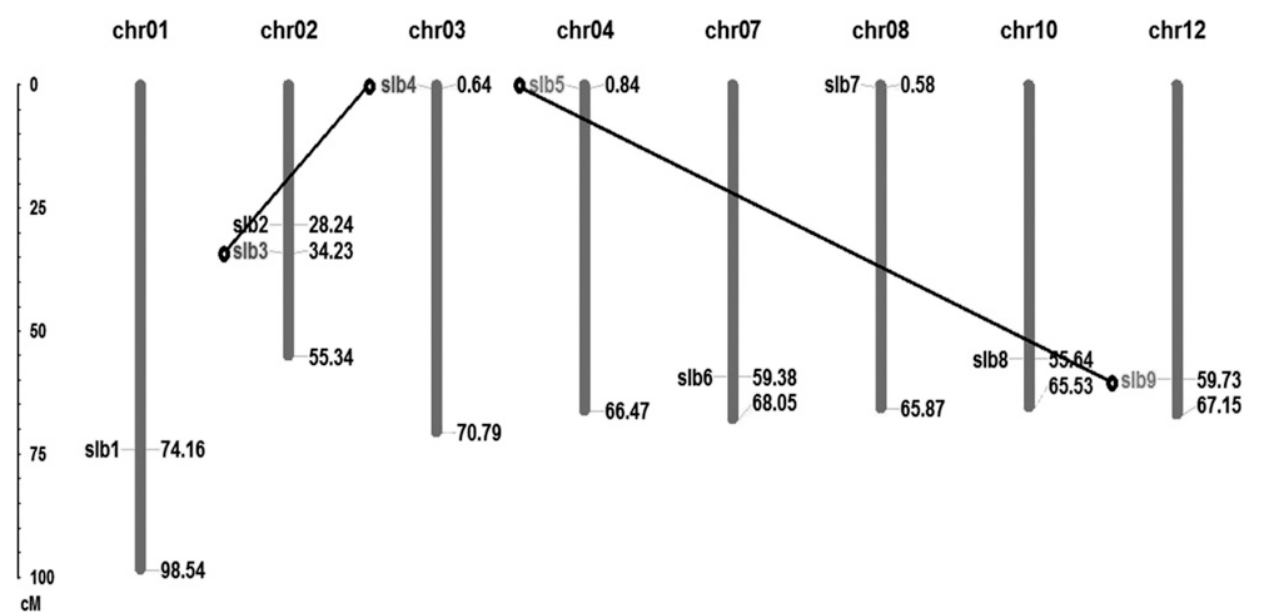

Fig. 6. Distribution of BRI1-EMS-suppressor (BES1) genes in Solanum lycopersicum BRI1-EMS-suppressor 1 (SLB) on eight tomato chromosomes. The nine SLB genes unevenly located on each conserved collinear blocks of the chromosomes. The chromosome numbers are marked above each chromosome. The BES1 genes present on duplicated chromosomal segments are connected by black lines between the two relevant chromosomes (chr)

degree by common abiotic stresses, including exposure to drought, low temperature, and salt (Fig. 5). In tomato, SLB2 may be associated with the drought response, as it exhibited a stable downregulation trend under drought conditions. Thus, we deduce that SLB2 cannot be resistant to drought stress. Moreover, genes such as SLB5, 6 may be associated with drought. It has been reported that Gossypium hirsutum BES1 mRNA (GenBank KP272000.1) showed high resistance to drought in drought environments after spraying BR (Wenkai et al., 2015). SLB9 likely contributes to cold stress endurance, as shown by its expression pattern, whereas other genes, such as SLB1, $-6,-7$, and -8 might be repressed by low-temperature treatments. However, no studies on the association of BES1 TFs with cold resistance are available in the literature. Nonetheless, as almost all of these genes were markedly upregulated after 6 and $24 \mathrm{~h}$ of salt treatment, BES1 TFs appear to play an important role in salt resistance in tomato. Previous work indicated that one BES1 gene, BZR1 (AT1G19350) of arabidopsis, is over-expressed under cold, drought, and salt treatments (Li et al., 2009). SLB5 and SLB9 are in the same group (Group III), as is AT1G19530, so they may have similar function as $A T 1 G 19530$. SLB2 was upregulated under cold and $12 \mathrm{~h}$ of salt treatment, and SLB9 showed specific expression under drought, salt, and cold treatments. Moreover, high levels of expression were detected under salt treatment compared with that under the other treatments (Fig. 5B). These findings suggest that BES1 TFs might be involved in stress-related regulatory networks, especially for salt stress.

In conclusion, this study is the first to comprehensively investigate BES1 TFs in tomato, and a preliminary evaluation of their expression profiles under various abiotic stresses was performed. We employed an improved method based on protein domain and phylogenetic analysis to classify BES1 proteins from tomato, arabidopsis, and chinese cabbage, and this approach will be important for research in other species. Nine SLB genes were mapped to the eight chromosomes of 12 chromosomes of tomato, and two duplicated SLB genes were identified. In addition, tomato BES1 TFs were found to be related to stress responses. In the future, additional experiments should be conducted to determine the specific biological functions of each of these genes. However, the identification of putative functional motifs and the evaluation of expression profiles provide useful information for further exploration of the biological functions of each putative BES1 gene.

\section{Literature Cited}

Artimo, P., M. Jonnalagedda, K. Arnold, D. Baratin, G. Csardi, E. Castro, S. Duvaud, V. Flegel, A. Fortier, E. Gasteiger, A. Grosdidier, C. Hernandez, V. Ioannidis, D. Kuznetsov, R. Liechti, S. Moretti, K. Mostaguir, N. Redaschi, G. Rossier, I. Xenarios, and H. Stockinger. 2012. ExPASy: SIB bioinformatics resource portal. Nucleic Acids Res. 40:597-603.

Bai, Y., W.B. Zhu, X.C. Hu, C.C. Sun, Y.L. Li, D.D. Wang, Q.H. Wang, G.L. Pei, Y.F. Zhang, A.G. Guo, H.X. Zhao, H.B. Lu, X.Q. $\mathrm{Mu}$, J.J. Hu, X.N. Zhou, and C.G. Xie. 2016. Genome-wide analysis of the bZIP gene family identifies two ABI5-Like bZIP transcription factors, BrABI5a and BrABI5b, as positive modulators of ABA signalling in chinese cabbage. PLoS One 11(7):1-19.

Baloglu, M.C., V. Eldem, M. Hajyzadeh, and T. Unver. 2014. Genomewide analysis of the bZIP transcription factors in cucumber. PLoS One 9(4):e96014.

Bo, H., J. Jin, A. Guo, H. Zhang, J. Luo, and G. Gao. 2015. GSDS 2.0: An upgraded gene feature visualization server. Bioinformatics 31:1296-1297.

Broun, P. 2004. Transcription factors as tools for metabolic engineering in plants. Curr. Opin. Plant Biol. 7:202-209.

Center for Bioinformatics. 2015. Gene structure display server. 17 May 2017. <http://gsds.cbi.pku.edu.cn/>.

Century, K., T.L. Reuber, and O.J. Ratcliffe. 2008. Regulating the regulators: The future prospects for transcription-factor-based agricultural biotechnology products. Plant Physiol. 147:20-29.

Chen, L., W. Hu, S. Tan, M. Wang, Z. Ma, S. Zhou, X. Deng, Y. Zhang, C. Huang, G. Yang, and G. He. 2012. Genome-wide identification and analysis of MAPK and MAPKK gene families in Brachypodium distachyon. PLoS One 7(10):e46744.

Chen, Y., M. Li, X. Wu, Y. Huang, J. Ma, and A. Xiong. 2015. Genomewide analysis of basic helix-loop-helix family transcription factors and their role in responses to abiotic stress in carrot. Mol. Breed. 35:125.

Cheng, F., S. Liu, J. Wu, L. Fang, S. Sun, B. Liu, P. Li, W. Hua, and X. Wang. 2011. BRAD, the genetics and genomics database for Brassica plants. BMC Plant Biol. 11(1):136.

Crooks, G.E., G. Hon, J.M. Chandonia, and S.E. Brenner. 2004. WebLogo: A sequence logo generator. Genome Res. 14:11881190.

Cui, L., K. Feng, M. Wang, M. Wang, P. Deng, W. Song, and X. Nie. 2016. Genome-wide identification, phylogeny and expression analysis of AP2/ERF transcription factors family in Brachypodium distachyon. BMC Genomics 17(1):636.

Energy's Joint Genome Institute. 1997. Phytozome. 21 June 2017. $<$ https://phytozome.jgi.doe.gov/pz/portal.html $>$.

Espinosa-Ruiz, A., C. Martinez, M. Lucas, N. Fabregas, N. Bosch, A. Cano-Delgado, and S. Prat. 2017. Topless mediates brassinosteroid control of shoot boundaries and root meristem development in Arabidopsis thaliana. Development 144:1619-1628. 
Fujioka, S. and T. Yokota. 2003. Biosynthesis and metabolism of brassinosteroids. Annu. Rev. Plant Biol. 54:137-164.

Gene Denovo. 2015. Omicshare. 8 May 2017. <http://www.omicshare. com/tools/Home/Soft/heatmap $>$.

Guo, R., H. Qian, W. Shen, L. Liu, M. Zhang, C. Cai, Y. Zhao, J. Qiao, and Q. Wang. 2013. BZR1 and BES1 participate in regulation of glucosinolate biosynthesis by brassinosteroids in Arabidopsis. J. Expt. Bot. 64:2401-2412.

Hoagland, D.R. and D.I. Arnon. 1937. The water-culture method for growing plants without soil. California Agr. Expt. Sta. Circ. 347.

Huala, E., A. Dickerman, M. Garcia-Hernandez, D. Weems, L. Reiser, F. LaFond, D. Hanley, D. Kiphart, J. Zhuang, W. Huang, L. Mueller, D. Bhattacharyya, D. Bhaya, B. Sobral, B. Beavis, C. Somerville, and S.Y. Rhee. 2001. The Arabidopsis information resource (TAIR): A comprehensive database and web-based information retrieval, analysis, and visualization system for a model plant. Nucleic Acids Res. 29:102-105.

International Solanaceae Initiative. 2015. The Sol Genomics Network. 2 Jan. 2017. <https://sgn.cornell.edu/search/locus>.

Jie, W., J. Cui, and H. Zhu. 2013. Suppression of type I collagen in human scleral fibroblaststreated with extremely low-frequency electromagnetic fields. Mol. Vis. 19:885-893.

Jin, J.P., F. Tian, D.C. Yang, Y.Q. Meng, L. Kong, J.C. Luo, and G. Gao. 2017. PlantTFDB 4.0: Toward a central hub for transcription factors and regulatory interactions in plants. Nucleic Acids Res. 45:1040-1045.

Larkin, M.A., G. Blackshields, N.P. Brown, R. Chenna, P.A. McGettigan, H. McWilliam, F. Valentin, I.M. Wallace, A. Wilm, R. Lopez, J.D. Thompson, T.J. Gibson, and D.G. Higgins. 2007. Clustal W and Clustal X version 2.0. Bioinformatics 23:2947-2948.

Li, D., F. Fu, H. Zhang, and F. Song. 2015. Genome-wide systematic characterization of the bZIP transcriptional factor family in tomato (Solanum lycopersicum L.). BMC Genomics 16(1):771.

Li, J. and J. Chory. 1999. Brassinosteroid actions in plants. J. Expt. Bot. 50:275-282.

Li, L., X. Yu, A. Thompson, M. Guo, S. Yoshida, T. Asami, J. Chory, and Y. Yin. 2009. Arabidopsis MYB30 is a direct target of BES1 and cooperates with BES1 to regulate brassinosteroid-induced gene expression. Plant J. 58:275-286.

Magadum, S., U. Banerjee, P. Murugan, D. Gangapur, and R. Ravikesavan. 2013. Gene duplication as a major force in evolution. J. Genet. 92:155-161.

National Institutes of Health. 2015. Mutiple Em for motif elicitation. 15 May 2017. <http://meme-suite.org/tools/meme>.

Pfaffl, M.W. 2001. A new mathematical model for relative quantification in real-time RT-PCR. Nucleic Acids Res. 29:e45-e51.
Phoenix Bioinformatics Corporation. 2013. The Arabidopsis information resource. 20 Apr. 2017. <http://www.arabidopsis.org/servlets/ Search?action=new_search\&type $=$ gene $>$.

Rio, D.C., M. Ares, Jr., G.J. Hannon, and T.W. Nilsen. 2010. Purification of RNA using TRIzol (TRI reagent)Cold Spring Harb. Protoc.20106pdb.prot5439.

Ryu, H., K. Kim, H. Cho, and I. Hwang. 2010. Predominant actions of cytosolic BSU1 and nuclear BIN2 regulate subcellular localization of BES1 in brassinosteroid signaling. Mol. Cells 29:291-296.

Salazarhenao, J.E., R. Lehner, I. Betegónputze, J. Vilarrasablasi, and A.I. Cañodelgado. 2016. BES1 regulates the localization of the brassinosteroid receptor BRL3 within the provascular tissue of the Arabidopsis primary root. J. Expt. Bot. 67:4951.

Song, H., P. Wang, Z. Nan, and X. Wang. 2014. The WRKY transcription factor genes in Lotus japonicus. Intl. J. Genomics 2014:420128.

Tamura, K., D. Peterson, N. Peterson, G. Stecher, M. Nei, and S. Kumar. 2011. MEGA5: Molecular evolutionary genetics analysis using maximum likelihood, evolutionary distance, and maximum parsimony methods. Mol. Biol. Evol. 28:2731-2739.

Wang, C., W. Duan, A.R. Riquicho, Z. Jing, T. Liu, X. Hou, and Y. Li. 2015. Genome-wide survey and expression analysis of the PUB family in chinese cabbage (Brassica rapa ssp. pekinesis). Mol. Genet. Genomics 290:2241-2260.

Wei, G., L. Long, X. Tian, J. Jin, H. Liu, H. Zhang, F. Xu, and C. Song. 2016. Genome-wide identification and expression analysis of stressassociated proteins (SAPs) containing A20/AN1 zinc finger in cotton. Mol. Genet. Genomics 291:1-15.

Wei, X., L. Wang, J. Yu, Y. Zhang, D. Li, and X. Zhang. 2015. Genome-wide identification and analysis of the MADS-box gene family in sesame. Gene 569:66-76.

Wenkai, A.N., D. Chang, and F. Zhang. 2015. Expression characteristics of transcription factor BES1/BZR1 of cotton seedling in response to brassinosteroid under drought stress. Acta Botanica Boreali-Occidentalia Sinica 35:1311.

Wu, P., X. Song, Z. Wang, W. Duan, R. Hu, W. Wang, Y. Li, and X. Hou. 2016. Genome-wide analysis of the BES1 transcription factor family in chinese cabbage (Brassica rapa ssp. pekinensis). Plant Growth Regulat. 80:291-301.

Yin, Y., D. Vafeados, Y. Tao, S. Yoshida, T. Asami, and J. Chory. 2005. A new class of transcription factors mediates brassinosteroidregulated gene expression in Arabidopsis. Cell 120:249-259.

Yin, Y., Z.Y. Wang, S. Mora-Garcia, J. Li, S. Yoshida, T. Asami, and J. Chory. 2002. BES1 accumulates in the nucleus in response to brassinosteroids to regulate gene expression and promote stem elongation. Cell 109(2):181.

Zhao, T., D. Liang, P. Wang, J. Liu, and F. Ma. 2012. Genome-wide analysis and expression profiling of the DREB transcription factor gene family in Malus under abiotic stress. Mol. Genet. Genomics 287:423-436. 


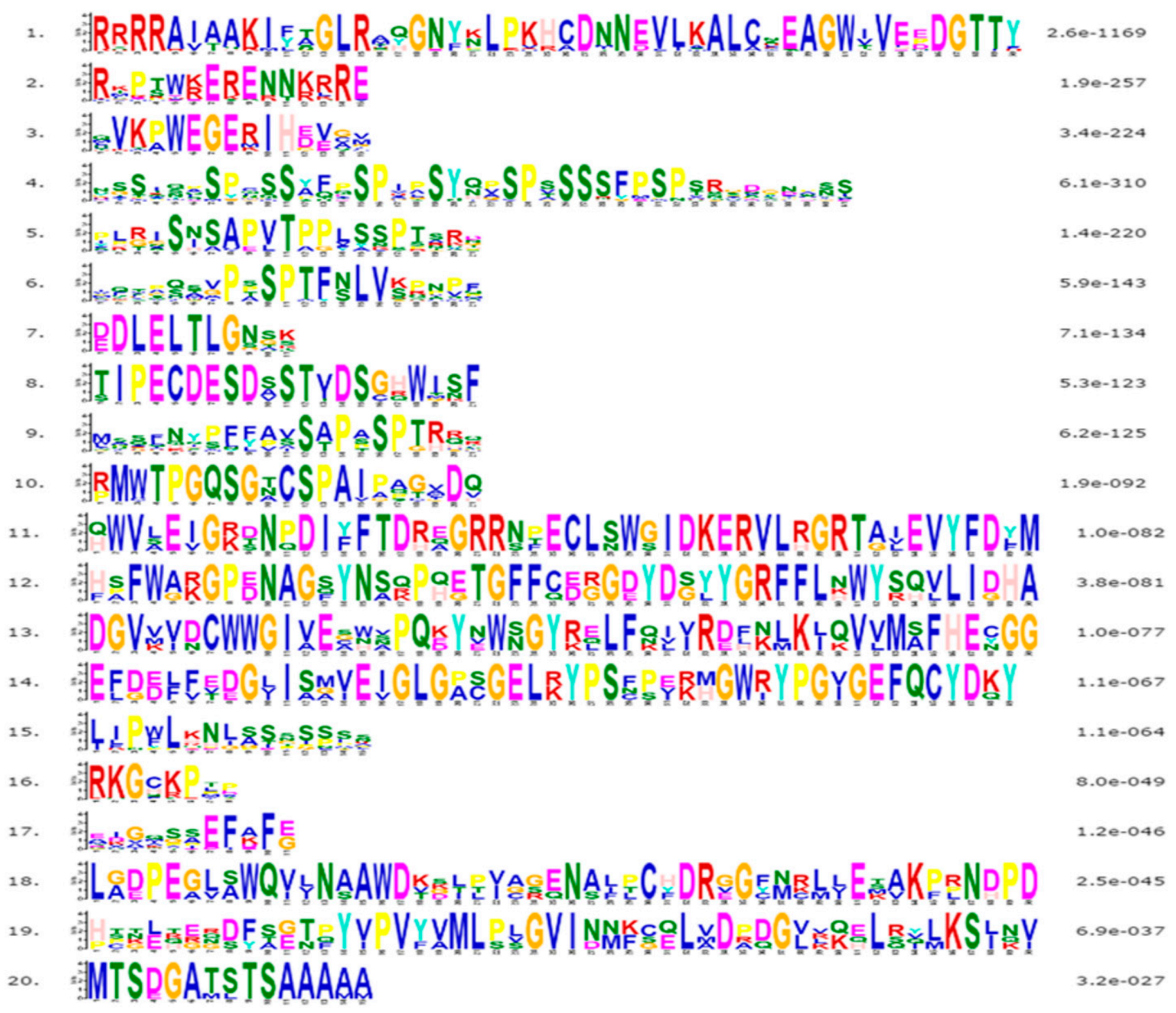

Supplemental Fig. 1. Sequence logos of BRI1-EMS-suppressor 1 domains in tomato. The overall height of the stack indicates the level of sequence conservation. The height of residues within the stack indicates the relative frequency of each residue at that position.

Supplemental Table 1. The primers were applied for quantitative real-time polymerase chain reaction amplification in this study.

\begin{tabular}{lll}
\hline Gene name & \multicolumn{1}{c}{ Forward primer $\left(5^{\prime}-3^{\prime}\right)$} & Reverse primer $\left(5^{\prime}-3^{\prime}\right)$ \\
\hline SLB1 & AGGCTCAGTCCCCTTCTCAT & TCGGAAACTAGTCAACCACAAA \\
SLB2 & TGGGAATCCCTCTCCAGGGT & AGGCGGCATCAGACTCATCA \\
SLB3 & CACGAGGCTACCGACATGGA & TCTTCAACAATCCAACCAGCCTCT \\
SLB4 & GTGAAGCCATGGGAAGGTGA & GCGAGCTAGCCTAGACATCA \\
SLB5 & TGTGGGAAGGTGGAGGGTTG & CCCTTTCCCTCCATGATGGCT \\
SLB6 & TGGACAGTTGAGCCCGATGG & AAGAGGAAGCAGGGCTTGGG \\
SLB7 & ACGTTCTCACCCTGGCAAGT & AGCCATCCTGGTTCGTTGGG \\
SLB8 & GGGGGTAGGGGATTGATTACAA & ACGTTTGCGTTGGAGGAATC \\
SLB9 & GTGCCGGAGTTGGTTTACCG & GCAGCTACAGCTCTCCTCCT \\
\hline
\end{tabular}

SLB = Solanum lycopersicum BRI1-EMS-suppressor 1. 